\title{
RAPID, AUTOMATED MEASUREMENT OF DIELECTROPHORETIC FORCES USING DEP-ACTIVATED MICROWELLS
}

\section{Lionel M. Broche ${ }^{{ }^{*}}$, Kai F. Hoettges ${ }^{1}$, Stephen L. Ogin ${ }^{1}$, George E. N. Kass ${ }^{2^{* *}}$, Michael P. Hughes ${ }^{1 * *}$}

${ }^{1}$ Faculty of Engineering and Physical Sciences and ${ }^{2}$ Faculty of Health and Medical Sciences, University of Surrey, Guildford, Surrey GU2 7XH, UK

*Current address:

**Current address:

***Corresponding author: email m.hughes@surrey.ac.uk

\begin{abstract}
Dielectrophoresis (DEP) is a physical effect that generates a force on a polarisable particles experiencing a non-homogeneous electric field. It has been shown that this effect depends on the electrical properties of that particle, i.e. the electrical permittivity and conductivity. In the past, DEP-based techniques were applied to measure the electric properties of one or several cells at a time. The results showed that the measure of electrical properties by DEP is very sensitive to noise. However, further improvements are possible by generating more information from the experiments: this paper presents a rapid automated system that measures the DEP spectrum from a large population of cells with a low level of noise using the microwell electrodes, and a method of analysis that provides additional information about the electrical properties of the cells and a new theoretical approach was developed to obtain accurate, bias-free results in under five minutes.
\end{abstract}




\section{Introduction}

Dielectrophoresis (DEP) is a physical phenomenon occurring wherever a polarisable particle is under the influence of an inhomogeneous electric field. According to its electrical properties, the particle moves along the direction of the electric field gradient in the direction of an either increasing or decreasing gradient: this behaviour has been defined as positive and negative DEP respectively. DEP can move particles over distances from a few micrometers to several millimetres by the use of appropriately designed electrodes. Measurement of the DEP force as a function of frequency allows extraction of cellular electrophysiological data, and has been shown to be useful in a range of settings from analysis of cancer cells [1-3] to cellular apoptosis [4,5] and the action of drugs on bacteria [6,7].

The theory behind DEP has been developed extensively since its discovery [8-11]. As the charges on a dipole experience Coulomb forces under the influence of an electric field, so will charges around a sphere experience similar forces under the same conditions. The charge distribution around a sphere due to the electric field can be approximated at the first order by a dipole-like distribution. The analytical expression of the magnitude of this equivalent dipole is expressed by a frequency-dependent factor called the 'Clausius-Mossotti factor' (CMF); its representation along with the frequency is often referred to as the 'DEP spectrum'. The force exerted on the particle by the electric field can be approximated by the interaction between the dipole described by the CMF and the electric field. This has been widely developed in the literature cited above and the expression of the resulting force, called the 'DEP force', is commonly expressed as:

$$
\overrightarrow{F_{D E P}}=2 \pi a^{3} \varepsilon_{m} \varepsilon_{0} \operatorname{Re}(K(\omega)) \nabla \vec{E}^{2}
$$

Where $\overrightarrow{F_{D E P}}$ is the DEP force experienced by the particle, $a$ is the radius of the particle, $\varepsilon_{m}$ is the electric permittivity of the surrounding medium, $\varepsilon_{0}$ is the electric permittivity of the free space, $\omega$ is the frequency, $\vec{\nabla} \vec{E}^{2}$ is the gradient of the electric field squared, Re is the real part operator and $K(\omega)$ is the CMF that describes the magnitude of the particle's equivalent dipole. The CMF itself can have several 
analytical expressions according to particle geometry; the most common geometries are the homogeneous sphere and the shelled sphere, the last one describing a sphere surrounded by a layer, and thus useful for modelling the cellular cytoplasm and plasma membrane. The homogeneous sphere model gives the following expression of the CMF [12]:

$$
K_{\text {sphere }}=\frac{\varepsilon_{c}^{*}-\varepsilon_{m}^{*}}{\varepsilon_{c}^{*}+2 \cdot \varepsilon_{m}^{*}}
$$

where $\varepsilon_{c}^{*}=\varepsilon_{c}+j . \sigma_{c} / \omega$ and $\varepsilon_{m}^{*}=\varepsilon_{m}+j . \sigma_{m} / \omega$. Here, $j, \varepsilon_{c}, \sigma_{c}, \varepsilon_{m}$, and $\sigma_{m}$ are the complex number, the sphere relative permittivity and conductivity and the surrounding medium relative permittivity and conductivity respectively. From this model it is possible to find the CMF of a shelled sphere of inner radius $a$ and layer thickness $\delta[13]$ :

$$
\varepsilon_{\text {eff }}^{*}=\varepsilon_{m}^{*} \frac{\alpha+2 . K_{\text {sphere }}}{\alpha-K_{\text {sphere }}} ; \quad K_{\text {shell }}=\frac{\varepsilon_{\text {eff }}^{*}-\varepsilon_{S}^{*}}{\varepsilon_{\text {eff }}^{*}+2 . \varepsilon_{S}^{*}}
$$

where $\varepsilon_{m}{ }^{*}$ is now the complex permittivity of the membrane, $\varepsilon_{S}{ }^{*}$ the complex permittivity of the suspension medium and $\alpha=(1+\delta / a)^{3}$. Equation 3 is known as the 'shell model' and is a model commonly used to approximate the behaviour of a cell experiencing DEP.

A number of methods have been employed to determine the DEP spectrum of a population of particles in suspension. Cell counting techniques consists of counting the number of cells collected at the surface of an electrode after a given time $[14,15]$ Since the motion of the entire population is examined over a wide frequency range, this approach offers the advantage of allowing insight into both membrane and cytoplasm, and can also be used to distinguish between different populations with different cytoplasmic conductivities $[4,15]$ It only requires a microscope, an electrode and a signal generator. Another more common technique measures the DEP force by 
monitoring the displacement of cells at different electric field frequencies with a camera and a tracking algorithm. The different positions of the cell provide the speed, and at the micron-scale the viscosity of water is so high that the speed is proportional to the force exerted, i.e. the DEP force. Then, by comparing the model to the data, the electric properties are retrieved [16-18]. Several laboratories have used this approach to demonstrate the feasibility of the measure of cell properties by DEP, which can be an interesting alternative to the more complex and expensive patch-clamp method.

In practice, variation between cells means that a large number of them must be measured in order to obtain a statistically significant result, and this is not always feasible with the methods described above because of the limitations of the tracking algorithm and visibility. In addition, planar electrodes also create very local electric fields, so that cell migration is very fast at the vicinity of the electrodes but is much slower everywhere else. Hence, the data collection for a 20 points spectrum can take up to an hour per sample. Since eukaryotic cells contain highly ionic cytoplasm and are suspended in low-conductivity solutions during the measure, there is a risk that ion exchange across the cellular membrane leads to a reduction in cytoplasm conductivity during the experiment, thereby creating artefacts in the results.

The define problem can be partly solved by using a 3 dimensional electrode such as a microwell electrode $[19,20]$. The electrical field generated by this structure extends far enough from the edges to provide detectable signal after a 1s application of the electric field making the acquisition much faster. It also takes advantage of a larger volume than in the planar electrode configuration, so the result is an average over several thousand cells, which makes the result more statistically significant for the measurement of a sample. The experimental time can be reduced further by automating the process. In this paper, we present a device developed for cell handling and data acquisition for DEP measures, providing a final acquisition time of 5 seconds per data point in the DEP spectrum, using a frequency band of $1 \mathrm{kHz}-20 \mathrm{MHz}$ and a signal amplitude of up to $20 \mathrm{Vpp}$. A typical spectrum acquisition lasts less than five minutes.

\section{DEVICE IMPLEMENTATION}




\subsection{Instrument controls and data acquisition}

The ideas described above were implemented in a machine that consisted of a fluid distribution unit that injected the sample to be analysed into the microwell, a mechanised unit that held the syringe and manipulated it, a valve that stopped the flow during the measure, an optical unit that took the measurements, and an electronic board connected to a PC that controlled the overall. The electrode used for generating the DEP force was a microwell encapsulated inside a plastic frame, as presented in Figure 1, and linked to the syringe by PTFE pipes (1/32' inner diameter) and connected to the signal generator (FG100, Digimess ${ }^{\circledR}$ ) and the oscilloscope (IDS710, Iso-tech), both controlled via serial port. A 2-position valve (Rheodyne 6-way valve distributor, model 7900-508-1) was used to stop the flow in the chip during the measurements. The microwell was monitored through a microscope equipped with a camera (16-bit Dolphin F145b, AVT). All devices were controlled from a PC using a software developed with MATLAB R2006a (The Mathworks, Natick, USA), which included the data acquisition and analysis.

The system operated in a programmed sequence. The resuspension unit drew the cell suspension into the well and stopped the flow during the measurement. The signal generator then activated at a frequency selected at random among a list given by the operator (in order to account for changes in environmental conditions over the duration of the experiment), and the camera recorded the change in light intensity across the well. The amplitude of the voltage was also monitored using the oscilloscope in order to detect and compensate for any loss of amplitude due to capacitive effects at high frequency. The field was applied for 5 seconds, which had been measured to be sufficient for an accurate measurement of the radial displacement of cells to be measured (see section 2.7). Once the measurement was completed, the electric field was switched off and the resuspension unit refreshed the cell suspension in the well. The procedure was then repeated until all frequency points had been examined. The entire procedure took less than 5 minutes to take 20 data points. A magnetic stirrer was used to prevent the cells from aggregating and settling and to keep the cell density homogeneous over the experiment time. 


\subsection{Theoretical simplifications}

The model of the CMF presented in equation 3 is limited for estimating the membrane properties when the membrane conductance is very low (typically below $0.1 \mathrm{~S} / \mathrm{m}$ ) [21]; the problem arises partly from the fact that the plasma membrane thickness of eukaryotic cells is difficult to measure, so the model has been modified for the present study. However, considering the common case that the plasma membrane thickness is much smaller than the cell radius, it is possible to perform a Taylor series of equation 3 , resulting in equation 4 :

$$
K=\frac{\varepsilon_{e f f}^{*}-\varepsilon_{3}^{*}}{\varepsilon_{e f f}^{*}+2 . \varepsilon_{3}^{*}} ; \quad \varepsilon_{e f f}^{*}=\frac{\varepsilon_{1}^{*} \varepsilon_{2}^{* 1}}{\varepsilon_{1}^{*}+\varepsilon_{2}^{* 1}}
$$

where $\varepsilon_{2}^{*^{\prime}}=\varepsilon_{2}^{*} \cdot a / \delta$ is the modified membrane complex permittivity. $\varepsilon_{2}^{* \prime}$ is proportional to the membrane properties by a factor $a / \delta$, which is typically several thousand. The simplification shown in equation 4 has the advantage of including the geometric factors into the unknown $\varepsilon_{2}^{*}$, making it unnecessary to measure the membrane thickness. It also pushes the value of $\varepsilon_{2}^{* 1}$ above the $0.1 \mathrm{~S} / \mathrm{m}$ limit observed by Gascoyne et al. [21] so that it makes the estimation of the membrane capacitance possible. This also diminishes the number of unknowns in the model so it is easier to compare it to the data by curve fitting. It can also be noticed that the measure of $\varepsilon_{2}^{*}$ ' provides a measure of $\varepsilon_{2}^{\prime}=\varepsilon_{2} a / \delta$ and $\sigma_{2}^{\prime}=\sigma_{2} a / \delta$, which are linked to the surface capacitance $\mathrm{C}$ and surface conductance $\mathrm{S}$ of the membrane as follows:

$$
\begin{aligned}
& C=\frac{\varepsilon_{2} \varepsilon_{0}}{\delta}=\frac{\varepsilon_{2}^{\prime}}{a} \varepsilon_{0} \\
& S=\frac{\sigma_{2}}{\delta}=\frac{\sigma_{2}^{\prime}}{a}
\end{aligned}
$$

So this approximation makes it possible to measure the membrane capacitance and conductance without using the membrane thickness. Finally, it is possible to build multishell models from this approximation and to obtain finer models that account for other layers.

\subsection{Microwell design}


The electrode geometry for the measurement of DEP behaviour used here is called a 'microwell' because of its well shape $[19,20]$. It consists in a multilayered structure of copper and polyimide sheets, with a $700 \mu \mathrm{m}$-diameter hole passing through the layers which forms the well where the sample sits. The layers of copper and polyimide are $75 \mu \mathrm{m}$ and $125 \mu \mathrm{m}$ thick, respectively. The exposed copper in the well has been electroplated with a gold layer to prevent oxidation and for better biocompatibility. The well is linked to two contact pads for the connection to the signal generator. A picture of the microwell chip used for the experiments is presented in Figure 2.

\subsection{Optics}

The optical system consists of a Zeiss Photomicroscope II connected to an AVT Dolphin F-145b camera. The capsule with the microwell is fixed on the microscope plate so that it cannot move during the experiment. The cell activity is observed by regular image acquisitions at the rate of 2 to 5 frames per second, with a resolution of $800 \times 600$, coded on 12-bit in greyscale. The data collected is sent to the PC via a Firewire connection. In order to ensure that the motion of cells across the entire chamber is monitored, a lens is added to the optical path to set the camera's focal point to infinity so that the level of light detected by one pixel gives a measure of the absorbance along the beam path.

\subsection{Samples}

In order to assess the significance of the measure made by the system, a test of the machine was performed using yeast cells. This choice was made because the DEP spectrum of yeasts has been widely measured in previous work [12,22-24] including that from our laboratory (REFS), allowing it to be used as a reference. The yeast cells (S. cerevisae, Allinson) were cultivated in suspensions of YPD Broth (Y1375, SigmaAldrich) at a concentration of 50 grams per litre of deionised water previously autoclaved at $121^{\circ} \mathrm{C}$ for 20 minutes. When cold, YPD broth was inoculated with yeast, shaken $5 \mathrm{~min}$ amd incubated at $36^{\circ} \mathrm{C}$ for $18 \mathrm{H}$. An isotonic low conductive media was prepared out of distilled water with $51 \mathrm{~g} / \mathrm{L} \mathrm{D-mannitol} \mathrm{and} \mathrm{a} \mathrm{small} \mathrm{amount}$ of phosphate buffered saline to adjust the conductivity to $3 \mathrm{mS} . \mathrm{m}^{-1}$. Prior to experimentation, the cells were centrifuged three times at $600 \mathrm{x} \mathrm{g}$ for $150 \mathrm{~s}$ and resuspended in the low-conductivity medium. The yeast suspension was then injected 
into the system. The survival rate was assessed once using cell count with Trypan blue after the experiment finished: $95 \%$ of the cells were found to survive after 20 min of continuous experimentation.

\section{6. data acquisition}

The DEP force was measured on the sample at 13 different frequencies in random order between $1 \mathrm{kHz}$ and $20 \mathrm{Mhz}$, i.e. 3 points per decade. For each frequency the acquisition lasted 5 seconds with the application of a $15 \mathrm{~V}$ peak-to-peak AC signal at the electrodes. With such parameters, one full spectrum took less than 5 minutes to measure. In order to check the repeatability, 14 spectra were acquired for comparison. One of them is shown in Figure 3 together with a best-fit spectrum constructed using the simplified shell model outlined in Equation 4, adding an extra shell. The fit was performed with Matlab, using the fit function from the fitting toolbox with the options 'Robust' using the Gauss-Newton algorithm. The results are presented in Table 1 along with the results from previous works found in the literature. The curve fitting algorithm also provided a $95 \%$ confidence bound which are reported between brackets on Table 1.

\subsection{Image processing}

Data processing is a critical point in this system and needs particular attention. Unlike a method which relies on the direct observations of cells, the DEP force cannot be measured directly from the images acquired from the well system so a model has been built to predict the images according to the DEP force.

The electric field in the electrode has been determined analytically and the corresponding expression of the DEP force has been deduced from it, using equation 1. The physical laws relevant to the study of the electric field concern the equations of an $\mathrm{AC}$ field for a frequency band of $1 \mathrm{kHz}$ to $20 \mathrm{MHz}$. The minimum corresponding electric wavelength is then $\lambda_{\max }=$ maximum frequency $/$ speed of light $\approx 0.3 \mathrm{~m}$, which is 100 times larger than the radius of the microwell. The quasi-static approximation can then be used to describe the electric field, the material permittivity $\varepsilon$ being changed into a complex permittivity $\varepsilon^{\prime}=\varepsilon-j \sigma / \omega$ where $\sigma$ is the material conductivity and $\omega$ the frequency, $j=\sqrt{-1}$. The electric potential is then found by 
solving the Laplace equation in the quasi-static approximation in the cylindrical geometry, the boundary condition being the voltage on the wall of the well. This technique consists in using the Fourier transformation of the electric potential at the surface of the microwell and solving the electric field for each harmonic. We used here a linear approximation of the wall potential. In the case of the microwell geometry, this provides the following electric potential:

$$
\left\{\begin{array}{l}
V(r, z)=\sum_{n} \frac{v_{n} \cos \left(\omega_{n z} z\right) I_{0}\left(\omega_{n z} r\right)}{I_{0}\left(\omega_{n z} R\right)} \\
v_{n}=\sqrt{\frac{2 V_{0}}{\pi}} \frac{2 \cos \left(\omega_{n z} L_{e} / 2\right)-\omega_{z} L_{i} \sin \left(\omega_{n z} L_{e}+\omega_{n z} L_{i}\right)-2 \cos \left(\omega_{n z} L_{e} / 2+\omega_{n z} L_{i}\right)}{\omega_{n z}^{2} L_{i}}
\end{array}\right.
$$

where $L_{e}\left(L_{i}\right)$ is the thickness of the electrode (isolator) layers, $V_{0}$ is the amplitude of the AC signal, $I_{0}$ is the Bessel function of order $0, R$ is the radius of the well and $\omega_{z}$ is the spatial frequency over the z-axis defined by $\omega_{n z}=\pi n /\left(L_{e}+L_{i}\right), n$ being a positive integer. The cosine and sine terms come from the Fourier decomposition of the surface potential. From Equation 5, it is possible to derive the electric field and to find the DEP force field for a given particle, in the dilute limit approximation:

$$
\left\{\begin{array}{l}
\vec{E}=-\operatorname{grad}(V) \\
\vec{F}_{D E P}=2 \pi a^{3} \varepsilon \varepsilon_{0} K \vec{\nabla}\left(\vec{E}^{2}\right)
\end{array}\right.
$$

where $a, \varepsilon$ and $\varepsilon_{0}$ are the radius of the cell, the relative permittivity of the medium and the electric permittivity of vacuum.

The cells migration can be predicted from the law of diffusion under a force field. Using the DEP force field expressed in Equation 6 with the Equation 7 of diffusion at low Reynolds number provides the diffusion Equation 8 under a DEP force field:

$$
\frac{\partial c}{\partial t}=-\operatorname{div}\left(-D \vec{\nabla} c+c \vec{F}_{D E P} / 6 \pi \eta a\right)
$$




$$
\frac{\partial c}{\partial t}=D \nabla c-\frac{2 a^{2} \varepsilon \varepsilon_{0} K}{3 \eta} \operatorname{div}\left(c \vec{\nabla} \vec{E}^{2}\right)
$$

where $c$ is the concentration of particles in space and $\eta$ is the dynamic viscosity of water. Equation 8 cannot be solved analytically for the concentration $c$ because of the divergence term. However the concentration is initially homogeneous so if we consider the small-time approximation we obtain the following:

$$
\left.\frac{\partial c}{\partial t}\right|_{t \rightarrow 0}=-\frac{2 a^{2} \varepsilon \varepsilon_{0} K}{3 \eta} c_{0} \operatorname{div}\left(\vec{\nabla} \vec{E}^{2}\right)
$$

The limit of the small-time approximation has been measured empirically from the data and is $0.2 \mathrm{~s}$ to $1.5 \mathrm{~s}$ depending on the voltage and microwell dimensions.

Finally Equation 9 combined with the Beer-Lambert law of absorbance (Equation 10) provides the model Equation 11 used to analyse the image of the cells in the microwell:

$$
\begin{aligned}
& I=I_{0} \exp \left(-\alpha / Z \int c d z\right) \\
& K=\left.\frac{3 \eta Z}{2 a^{2} \varepsilon \varepsilon_{0} \alpha c_{0} J} \frac{\partial \ln \left(I / I_{0}\right)}{\partial t}\right|_{t \rightarrow 0}
\end{aligned}
$$

where $I$ is outward light flux measured, $I_{0}$ is the illumination flux, $\alpha$ is the light absorption coefficient of the particle and $Z$ is the length of the path of light, i.e. the thickness of the microwell. Equation 11 relates the CMF to the light intensity measured via a factor that remains constant over the experiment. It makes it possible to measure the CMF of the particles in the well by monitoring the evolution of the light flux passing through the well, and then by processing the images.

\section{RESULTS}


Figure 3 shows a spectrum obtained from an average of four experiments on yeast cells measured in the conditions cited above, the error bars indicating the standard deviation between the acquired spectra. The dashed curve that appears on the figure represents the average fit using the double shell model with thin membrane approximation on the yeast spectra. The difference between the fitted curve and the data from separate yeast spectra run at $5.1 \times 10^{8}$ cells $/ \mathrm{ml}$ provided an estimation of the error in the data and is presented in Figure 4. This histogram indicated a noise level under $5 \%$ for $45 \%$ of the data acquired. A significant part of this noise was due to the discrepancy between the data and the fit below $6 \mathrm{kHz}$, which is attributed to the effect of electrohydrodynamic flows, which are known to perturb DEP collection in that frequency range [25]. If this low-frequency part is removed from the histogram of the noise, then $60 \%$ of the data acquired has a level of noise under $5 \%$.

Table 1 presents the result of the curve fitting for the four yeast spectra considered above on the left column, and the values found in the literature on the right column. The values of the parameters obtained from the curve fitting applied to the dispersion curves had acceptable confidence bounds with regards to the literature, with the exception of the cytoplasm permittivity. The latter affects mostly the high-frequency end of the spectra, typically above $50 \mathrm{MHz}$, which could not be covered in our experiment since the frequency generator was limited to $20 \mathrm{MHz}$. Hence this problem was expected for the cytoplasm permittivity. The numerical values found for the other parameters of yeast cells fell into the bounds defined by the literature, even though the value of the membrane conductance is relatively high. However, the different measurements of this conductance found in the literature reports that it varies over a broad range of values, which indicate that this parameter is likely to be sensitive to the particular strain of yeast or the experimental conditions. The values found for the membrane capacitance and the wall capacitance and conductance gave correct orders of magnitudes compared with the values reported from the literature. The differences observed may have several causes. It may be explained by natural variations in the biological system used due to the culture conditions and yeast strains, in which case more experiments are needed using several strains of yeast in order to determine the variation due to this effect, or physiological changes of the yeast cells to adapt to the culture medium, which is difficult to control. 


\section{CONCLUSION}

This is the first system capable of collecting substantial data across a full DEP spectrum, allowing the determination of electrical properties of cytoplasm, membrane and wall of a relatively large population of cells under 5 minutes and with a high signal-to-noise ratio and a high inter-experimental reproducibility. The method presented can measure the membrane properties more accurately than by previous works using DEP because the thin membrane approximation pushes the signal above the minimum threshold defined in the literature. Hence, the error bars on the membrane conductance and capacitance are low enough to provide quantitative data. Such a system clearly demonstrates the potential DEP as an assay technology to determine cell electrophysiology and overcomes most problems previously associated with DEP based assays. The high degree of automation replaces the formally high cost and manpower associated with DEP assays with a simple and easy to use system that can be used as a routine tool in cell in many cell based applications.

\section{ACKNOWLEDGEMENTS}

This work was supported by the EPSRC, grant number GR/S85443/01. 


\section{References}

1. Gascoyne, Wang, et al. IEEE Trans. Ind. Appl._1997, 33, 670-8.

2. DeGasperis, Yang, et al. Biomedical Microdevices 1999, 2, 41-49.

3. Labeed, Coley, et al. . Biophys. J. 2003, 85, 2028-34.

4. Labeed, Coley, et al. . Biochim. Biophys. Acta 2006, 1760, 922-929.

5. Pethig and Talary. IET Nanobiotechnol. 2007, 1, 2-9.

6. Hoettges, Dale, et al.. Phys. Med. Biol. 2007, 52, 6001-6009.

7. Zhou, Markx et al.. Biochim. Biophys. Acta 1995, 1245, 85-93

8. Pohl and Pethig. J. Phys.E: Scientific Instruments 1977, 10. 193.

9. Wang, Huang, et al.. J. Phys. D: Appl.Phys. 1994, VOLUME? 1571-1574.

10. Jones. IEEE Eng. Med. Biol._2003, 22, 33 PAGES?.

11. Karttunen, Huang, et al.. Phys. Rev. E 2003, 67, 21403.

12. Huang, Holzel, et al.. Phys. Med. Biol. 1992, 37, 1499-1517.

13. Hanai, Koizumi and Irimajiri. Biophysics of Structure and Mechanism 1975, 1, 285-94

14. Pohl and Crane. Biophys. J 1971, 11, 711-727.

15. Broche, Labeed, et al. Physics in Medicine and Biology 2005, 50, 1-8.

16. Gascoyne, Huang, et al. Meas. Sci. Technol. 1992, 3, 439-445.

17. Pethig, Talary, et al. IEEE Engineering in Medicine and Biology Magazine 2003, $22,43$.

18. Pethig, Jakubek, et al. IEEE Proc.-Nanobiotechnol. 2005, 152, 189-193.

19. Huebner, Hoettges, et al. IEEE Proc.-Nanobiotechnol. 2005, 152.

20. Hoettges, Hubner, et al. Analytical Chemistry 2008, 80, 2063-2068.

21. Gascoyne, Becker, et al. Bioelectrochemistry and Bioenergetics 1995, 36, 115 125.

22. Hoettges, McDonnell, et al. Journal of Physics D: Applied Physics 2003, 36, 101104.

23. Kunze, Kell, et al. Single Molecules 2003, 3, 169-70.

24. Suehiro, Zhou, et al. IEEE Transactions on Industry Applications 2003, 39, 1514.

25. Castellanos, Ramos, et al. Journal of Physics D: Applied Physics 2003, 36, 2584.

26. Holzel Biophysical Journal 1997, 73, 1103.

27. Fatoyinbo, Hoettges, et al. Electrophoresis 2008, 29: 3-10.

28. Raicu, Raicu, et al. Biochim Biophys Acta 1996, 1274, 143-148. 


\section{FIGURES AND TABLES}

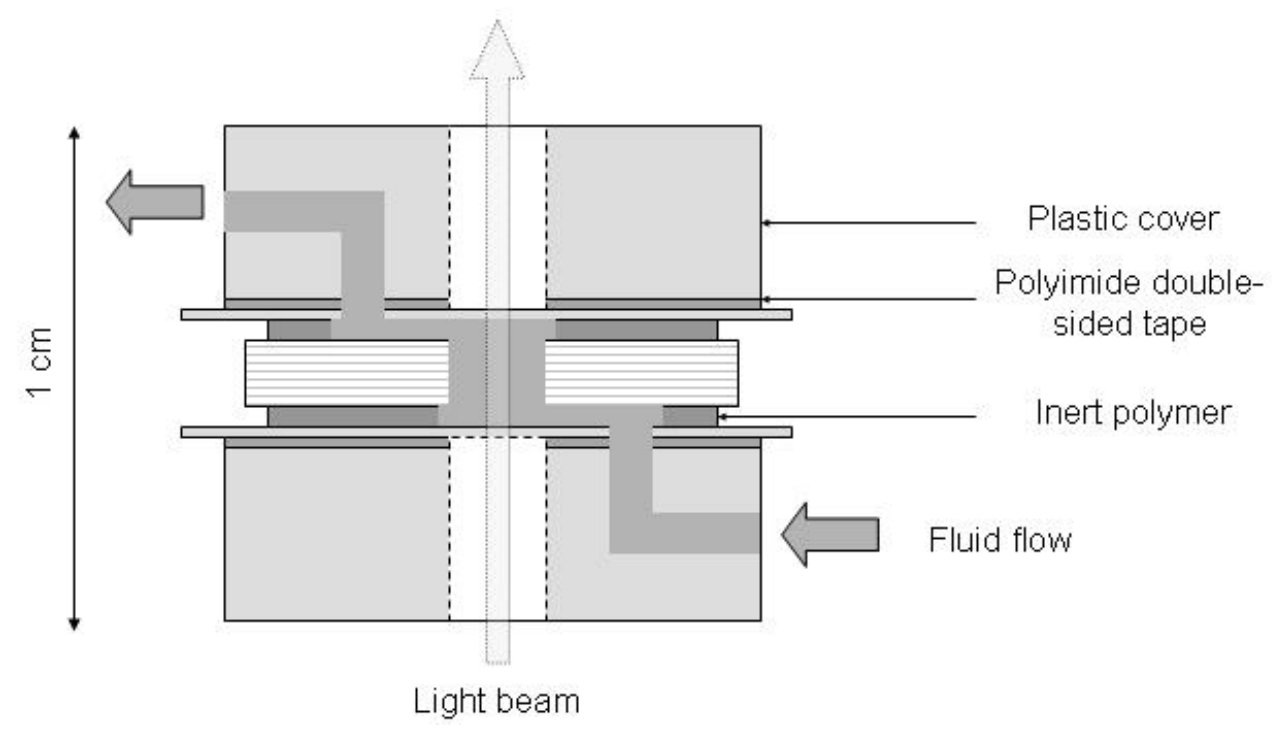

Figure 1: Scheme of an encapsulated microwell

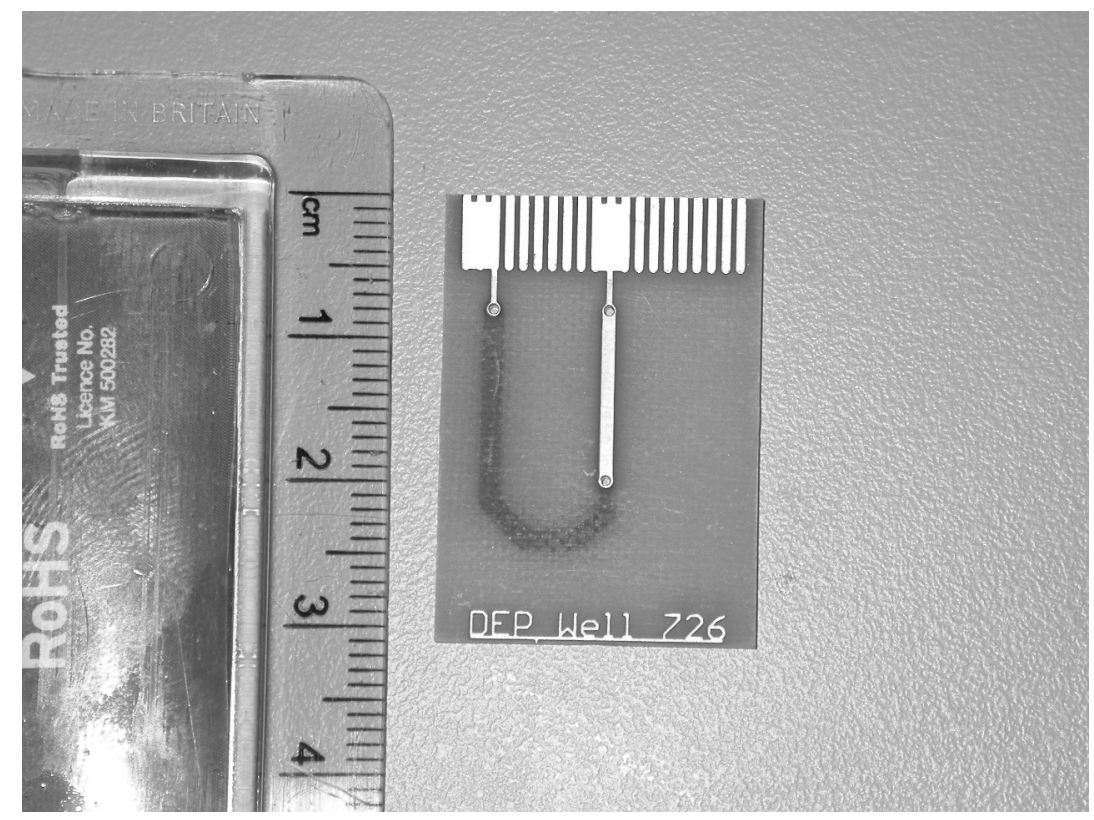

Figure 2: Microwell chip used for the experiments. 


\section{Comparison between an average spectrum of yeast cells}

and the corresponding fit

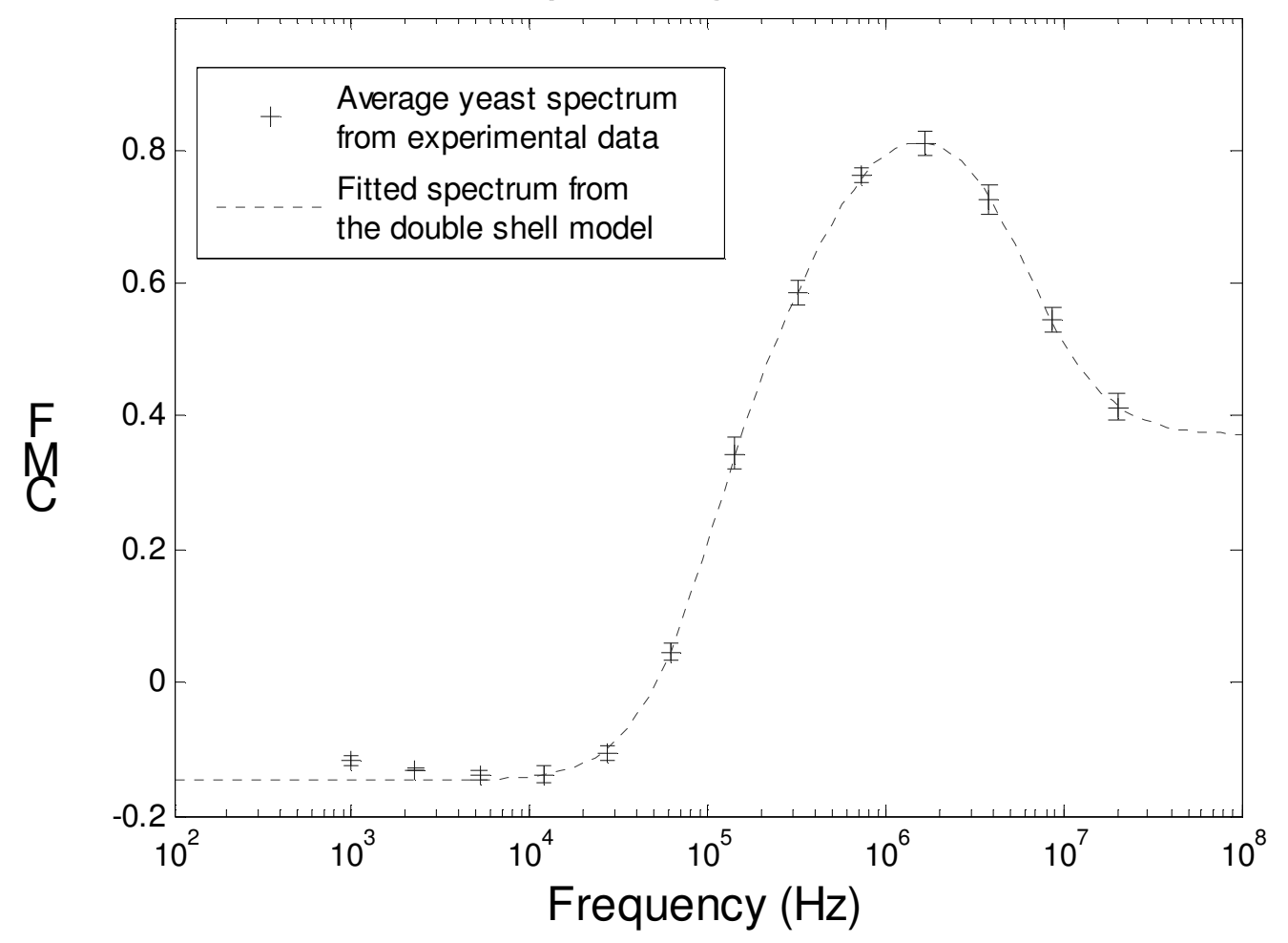

Figure 3: comparison between a yeast spectrum averaged from $\mathbf{4}$ different spectra measured with the machine and the average fit using the double shell model with the thin membrane approximation. Here, the value of $R^{2}$ is 0.9989 . 
Histogram of the noise in yeast spectra acquired by the machine

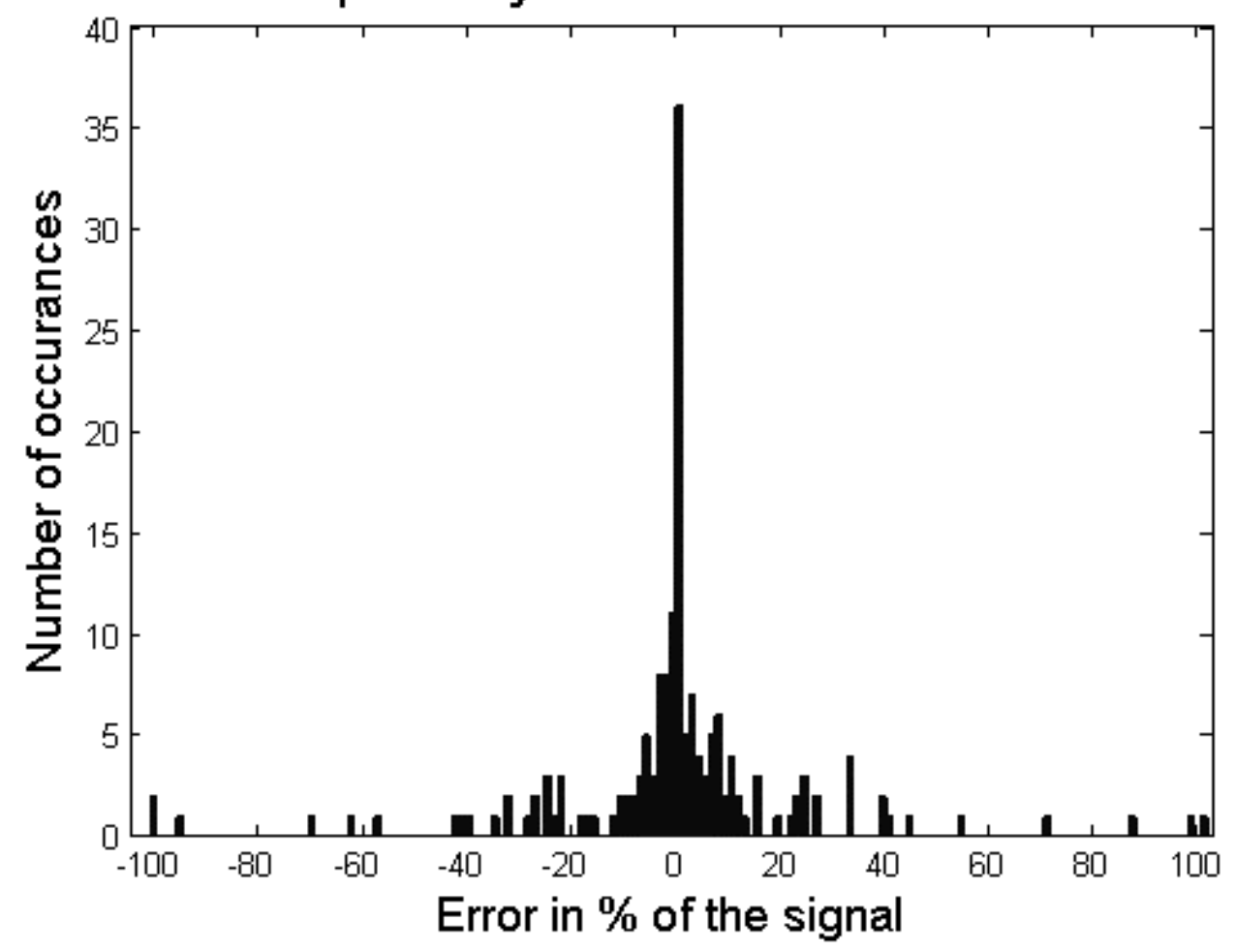

Figure 4: Histogram of the fit residues measured on the spectra of yeast cells. 


\begin{tabular}{|c|c|c|}
\hline & Obtained results & Published results \\
\hline $\begin{array}{c}\text { Cytoplasm } \\
\text { conductivity }\end{array}$ & $95 \pm 15 \mathrm{mS} / \mathrm{m}$ & $\begin{array}{c}550 \pm 50 \mathrm{mS} / \mathrm{m}[1] \\
140 \pm 40 \mathrm{mS} / \mathrm{m}^{2}[2]\end{array}$ \\
\hline $\begin{array}{c}\text { Cytoplasm } \\
\text { permittivity }\end{array}$ & $195 \pm 20$ & $50.6 \pm 2.1[3]$ \\
\hline $\begin{array}{c}\text { Membrane } \\
\text { capacitance }\end{array}$ & $0.66 \pm 0.3 \mu \mathrm{F} / \mathrm{cm}^{2}$ & $0.703 \pm 0.011 \mu \mathrm{F} / \mathrm{cm}^{2}[3]$ \\
\hline $\begin{array}{c}\text { Membrane } \\
\text { conductance }\end{array}$ & $1000 \pm 90 \mathrm{~S} / \mathrm{m}^{2}$ & $\mathbf{0 . 7 6} \mu \mathrm{F} / \mathrm{cm}^{2}[1]$ \\
\hline $\begin{array}{c}\text { Wall } \\
\text { conductivity }\end{array}$ & $8 \pm 2 \mu \mathrm{S} / \mathrm{cm}^{2}$ & $5.5 \mathrm{to} 50 \mathrm{~S} / \mathrm{m}^{2}[1]$ \\
\hline \hline $\begin{array}{c}\text { Wall } \\
\text { permittivity }\end{array}$ & $0.27 \pm 0.07 \mu \mathrm{F} / \mathrm{cm}^{2}$ & $\mathbf{6}$ to $240 \mu \mathrm{S} / \mathrm{cm}^{2}[1]$ \\
\hline
\end{tabular}

Table 1: experimental results compared with previously published data. A[26] (Holzel 1997); [2] (27 et al. 2008); [3] (Raicu et al. 1996). 